\title{
Ultrasound-guided percutaneous thrombin injection following iatrogenic femoral artery pseudoaneurysm: patient selection and perspectives
}

This article was published in the following Dove Press journal: Journal of Vascular Diagnostics and Interventions

\author{
Grant Sizemore' \\ Farhan Ayubi ${ }^{2}$ \\ Benjamin Clark' \\ Dwight Kellicut ${ }^{2}$ \\ 'General Surgery, Tripler Army \\ Medical Center, Honolulu, HI, USA; \\ ${ }^{2}$ Vascular Surgery, Tripler Army \\ Medical Center, Honolulu, HI, USA
}

\begin{abstract}
Ultrasound-guided percutaneous thrombin injection (UGTI) following iatrogenic femoral artery pseudoaneurysms has been the standard of care as first-line treatment at many institutions since its initial description by Liau et al in 1997. UGTI underwent rapid adoption given its significant advantages to patients including shorter procedure times, decreased discomfort, lower rates of recurrence, and the avoidance of surgical intervention in appropriate cases. Despite the availability of less-invasive approaches, through numerous head-to-head studies, UGTI has demonstrated clear benefit over ultrasound-guided compression in the treatment of iatrogenic femoral artery pseudoaneurysms. Although newer interventions such as percutaneous coil embolization have attracted attention for their superior ability to manage those patients with high-level anticoagulation or allergies to thrombin, at this time they do not provide an equal alternative to thrombin injection. In this review, we focus on issues related to the selection of patients who benefit from UGTI for the treatment of iatrogenic pseudoaneurysms.
\end{abstract}

Keywords: false aneurysm, endovascular complication, cardiac catheterization, interventional radiology, autologous thrombin

\section{Introduction}

Pseudoaneurysms can arise from percutaneous access to the arterial system if the artery is not adequately sealed once a sheath is removed. These aneurysms are deemed "false" as they contain no elements of the arterial wall, but rather have a wall composed of thrombus and the surrounding soft tissues. They differ from a hematoma, a simple collection of blood in an extravascular space, as pseudoaneurysms have a defined neck communicating with an arteriotomy. ${ }^{1}$

This complication is well described in the literature, ranging from $1 \%$ in diagnostic procedures to as high as $8 \%$ in therapeutic interventions. ${ }^{2,3}$ This significantly exceeds the standards for acceptable incidence as established by numerous societies (as low as $0.2 \%$ for diagnostic studies) over 20 years ago, ${ }^{4}$ but highlights a persistent issue with current arterial access techniques. There are several factors associated with the formation of pseudoaneurysms. According to the Contemporary Management of Post Catheterization Pseudoaneurysms by Webber et $\mathrm{al}^{5}$ the factors include the use of antiplatelet agents, anticoagulation, sheath size $>8 \mathrm{~F}$, age $>65$ years, obesity, poor postprocedural compression, simultaneous artery and vein catheterization, hypertension, peripheral vascular disease, hemodialysis, complex interventions, and low or high puncture sites.

In early studies, the reported rates were much lower $(0.05 \%-2.00 \%)$; however, these findings were retrospective and annotated only those complications which required
Correspondence: Grant Sizemore General Surgery Department, I Jarrett White Road, Tripler Army Medical Center, Honolulu, HI, 96859, USA Email grant.c.sizemore.mil@mail.mil 
surgical correction. Further prospective sonographic studies show an increased incidence $(1.1 \%-6.24 \%)$, but these studies evaluated only patients undergoing retrograde puncture of the artery with clinically suspected pseudoaneurysm or arteriovenous fistula. Further prospective studies which use routine duplex imaging show incidence as high as 7.7\%, as many small, asymptomatic aneurysms were identified. ${ }^{6}$ A more recent study has demonstrated an incidence of $2.9 \%$ in $\sim 500$ consecutive patients undergoing cardiac catheterization. ${ }^{7}$

\section{Diagnosis}

The presence of pain, swelling, or pulsatile mass is the standard presentation of a large pseudoaneurysm after catheterization. Other, rarer symptoms can include neuropathy, venous thrombosis, claudication, or critical limb ischemia secondary to mass effect from a large pseudoaneurysm. On examination, there may be a palpable, pulsatile mass with or without an underlying systolic bruit. Therefore, the presence of disproportionate pain or swelling at the site of arterial access, even in the absence of bruit, should initiate further workup. ${ }^{5}$

The gold standard for diagnosis of pseudoaneurysm is duplex ultrasound with a sensitivity of $94 \%$ and a specificity of $97 \%$. Contrast-enhanced computed tomography of the abdomen and pelvis may be of benefit if significant extension into the retroperitoneum is suspected during duplex ultrasound. ${ }^{8}$ Often, small iatrogenic pseudoaneurysms are asymptomatic, and those smaller than $1.8 \mathrm{~cm}$ have a high likelihood to spontaneously thrombose (although this should not preclude treatment for prevention of possible complications).

\section{History}

Up until the early 1990s, the standard of care for nearly all femoral pseudoaneurysms was surgical intervention. While very effective, surgical therapy is not without risks. Lumsden et $\mathrm{al}^{9}$ describes a $20 \%$ complication risk for surgically treated groin complications following percutaneous cardiac procedures. The search for a safer but equally efficacious method was begun.

Throughout the 1990s, there were multiple studies done evaluating the natural history of pseudoaneurysms that did not meet criteria for acute surgical intervention, culminating with the work of Toursarkissian et al. ${ }^{10}$ They demonstrated that the vast majority of stable pseudoaneurysms, as many as $89 \%$, can be managed with observation alone. The paper, however, failed to address those on anticoagulation, making these findings difficult to generalize to patients at greatest risk for pseudoaneurysm formation. Additionally, the study required a follow-up protocol with frequent visits and duplex ultrasound evaluation. Patients in the nonoperative arm underwent an average of 2.6 duplex scans after diagnosis compared to 1.4 for the operative group. ${ }^{10}$

At the same time that Toursarkissian et al ${ }^{10}$ were performing their work, several other groups were looking at conservative management strategies for femoral artery pseudoaneurysms. The technique of ultrasound-guided compression was first described and demonstrated to be viable and effective in 1991 by Fellmeth et al. ${ }^{11}$ It soon was adopted as the standard first-line treatment for iatrogenic femoral artery pseudoaneurysm with numerous other studies throughout the following decade further validating the method. While it is effective, including nearly $94 \%$ initial success in those not on anticoagulation and up to $73 \%$ in those on long-term anticoagulation, ${ }^{12}$ it can take considerable time, cause significant patient discomfort, and require more involved follow-up.

There are a number of factors associated with failure of direct compression, most notably the ongoing need for anticoagulation and the length of the aneurysm track $(<10 \mathrm{~mm})$ as described by Schaub et $\mathrm{al}^{13}$ in their series of 219 consecutive patient. Other factors evaluated included gender, volume, duration, Broca index (as defined by the formula: body weight in $\mathrm{kg} /[$ height in $\mathrm{cm}-100]$ ), and systemic hypertension, all of which failed to demonstrate significance. In their study, Schaub et $\mathrm{al}^{13}$ also addressed the utility in reapplication of compression dressings. In 128 (58\%) patients, the pseudoaneurysm was initially treated with the reapplication of a firm compression bandage for 24 hours. This treatment was successful in only $32 \%$ of patients. Further subgroup analysis found differences in success only with regard to size of the pseudoaneurysm ( $\geq 6 \mathrm{~cm}$ ) and anticoagulation. No differences were found with regard to gender, Broca index, or length of the pseudoaneurysm tract $(>10 \mathrm{~mm})$.

\section{Development of the thrombin method}

The first description of the use of thrombin for the treatment of pseudoaneurysms was by Cope and Zeit ${ }^{14}$ in a 1986 case report, but it was not until 1997 when Liau et al $^{15}$ produced a detailed description of the treatment of iatrogenic femoral artery pseudoaneurysm with percutaneous thrombin injection that the technique was widely adopted and eventually became the standard first-line modality for the management of this complication. In the initial paper, which included only 5 patients, they were able to demonstrate a simple, nonmorbid, and highly successful treatment without recurrence at a mean follow-up time of 11.6 months. Numerous studies 
performed throughout the ensuing decade demonstrated excellent success rates for ultrasound-guided percutaneous thrombin injection (UGTI) ranging from $93 \%$ to $100 \%$ with minimal recurrence $(6 \%-14 \%)$ and low incidence of complication $(1 \%-2 \%){ }^{16-20}$

There are two prospective randomized control trials that evaluate ultrasound-guided compression compared to UGTI for the treatment of iatrogenic femoral artery pseudoaneurysm. In 2004, Lonn et al ${ }^{21}$ evaluated 30 consecutive patients with iatrogenic pseudoaneurysms over a 22 month period. Exclusion criteria included: any indication for surgical intervention, local infection, previous exposure to bovine thrombin with a known allergy, or a pseudoaneurysm neck $>10 \mathrm{~mm}$. The patients were then randomized to either compression (using FemoStop II Plus compression device RadiMedical Systems, Uppsala, Sweden) or thrombin injection via standard technique. The two groups were matched for age, sex, etiology of pseudoaneurysm, and the use of antiplatelet drugs. The primary outcome measured was successful thrombosis of the pseudoaneurysm at 24 hours. Secondary outcomes measured the presence or absence of thrombosis at 48 hours, complications, and length of hospital stay. The study found successful thrombosis in all 15 patients in the thrombin injection arm at 24 hours compared with only two (13\%) in the compression arm during the same time period. Six patients $(40 \%)$ had successful thrombosis 48 hours after repeat treatment $(p<0.001)$. All remaining pseudoaneurysms were successfully treated with thrombin injection. There were no complications or significant differences in length of stay noted. ${ }^{15}$

A second study completed in 2006 by Liu et al evaluated 38 patients with iatrogenic pseudoaneurysms. Neither exclusion criteria nor age and sex distribution were stated. The patients were randomized into an UGTI or ultrasound-guided compression group. Patients were evaluated at 3 and 7 days, with primary outcomes measuring successful thrombosis at 72 hours. Secondary outcomes evaluated thrombosis at 7 days and change in volume of hematoma. The study found successful thrombosis in all 19 patients within the thrombin group and $13(68 \%)$ in those managed with ultrasoundguided compression at 3 days. They also reported complete thrombosis of all pseudoaneurysms at 7 days (risk ratio [RR]: 1.44; 95\% CI: $1.06-1.97) .{ }^{21}$

Further analysis in a 2013 Cochrane review merging data from the above studies, with notations made accepting the limitations of different study designs and measures, found that percutaneous thrombin injection was not significantly more effective than ultrasound-guided compression (RR: 2.81 ; 95\% CI: 0.44-18.13) based on the parameters measured. All told, however, UGTI has supplanted ultrasound-guided compression and operative intervention unless otherwise indicated as it is thought to provide more rapid thrombosis, decreased procedure time, reduced pain, and improved success. ${ }^{22}$

\section{Patient selection}

There are no consensus guidelines regarding patient selection for ideal management of femoral artery pseudoaneurysms. Certainly, variables such as size, flow, lumen, and patient anticoagulation status should be considered. ${ }^{22}$ Current data suggest that pseudoaneurysms smaller than $3 \mathrm{~cm}$ in diameter will thrombose spontaneously; however, this occurs at a mean of 23 days. ${ }^{10}$ Samuels et $\mathrm{al}^{23}$ found that the greatest predictor of spontaneous thrombosis was aneurysmal neck length. An aneurysm neck $>0.9 \mathrm{~cm}$ would spontaneously thrombose at 9 days vs 52 days for those with a shorter neck length. Watchful waiting was also noted to have, as expected, a higher average number of follow-up visits and duplex examinations (2.6 vs 1.4 for immediate intervention group) ${ }^{10}$ Consensus data have shown that reliable patients with asymptomatic pseudoaneurysms smaller than $2 \mathrm{~cm}$ in diameter may follow-up with frequent duplex assessment. Intervention is recommended in the remaining patients. ${ }^{1}$

Indications for immediate surgical repair regardless of size include the following: hemodynamic instability, active bleeding, an expanding mass, distal ischemia, overlying skin necrosis, infection, or neurological deficit. ${ }^{1}$ Also, contraindications exist to the treatment of femoral artery pseudoaneurysms with thrombin injection. Certainly, percutaneous thrombin injection should only be performed in patients who develop the pseudoaneurysm secondary to a catheterization procedure. Those that arise spontaneously should be highly suspicious for a mycotic pseudoaneurysm and thus should be managed via other measures. Additionally, pseudoaneurysms occurring at the anastomosis of a synthetic graft to native artery should be managed surgically. ${ }^{5}$ Relative contraindications to thrombin injection include a large aneurysm $(>5 \mathrm{~cm})$, wide neck $(>1.0 \mathrm{~cm})$, or failure of repeated ultrasound-guided treatments. ${ }^{1}$

\section{Recurrence and repeat intervention}

Conservative management (ie, ultrasound-guided compression alone) is not without risk of failure or recurrence, as demonstrated by Cox et $\mathrm{al}^{24}$ in their study of 100 consecutive patients. They found that those on systemic anticoagulation were significantly more likely to fail intervention with compression than similarly matched patients without anticoagula- 
tion (98\% vs $86 \%, p=0.019)$, further supporting the pursuit of thrombin injection.

The question remains then as to which subset of femoral artery pseudoaneurysms will be refractory to percutaneous thrombin injection. Sheiman and Mastromatteo ${ }^{25}$ evaluated 54 consecutive iatrogenic pseudoaneurysms of the common femoral artery and had complete thrombosis in 49 after a single injection. Of the five that failed, four obtained obliteration on ultrasound 10 minutes after injection but recurred within 24 hours (clinically suspected, sonographically confirmed) while in the fifth only partial thrombosis was obtained despite repeated injection. All were referred for surgical management, and four out of five were found to have a laceration at the arteriotomy site of at least $0.8 \mathrm{~mm}(24 \mathrm{~F})$. There was no obvious laceration on ultrasound, even in retrospective review. One patient was found to have an infection at the common femoral artery site requiring bypass. The aneurysms that failed conservative management had no significant difference in size, amount of thrombin used, neck length, or size of vascular access.

There exists no contraindication to repeated injection; however, some authors warn that failure despite repeat injections or early failure within 24 hours should be viewed with suspicion and be potential selection criteria for operative management. No other studies reviewed discussed further criteria for potential failure of UGTI.

\section{Alternatives}

Alternative techniques exist to those discussed in detail above and they include para-aneurysmal saline injection, use of autologous thrombin, coil embolization, and placement of covered vascular stents. In 2003, Gehling et $\mathrm{al}^{26}$ demonstrated success in a small case series (6 patients) of femoral artery pseudoaneurysms managed with para-aneurysmal injection of saline $(52 \pm 33 \mathrm{~mL}$ of physiologic saline) with good success. This was followed up by a series of 64 consecutive patients who showed $92.2 \%$ success via similar technique (all failures were managed successfully with direct compression or thrombin injection), ${ }^{27}$ thus demonstrating a cost-effective, safe, minimally invasive, fast, and effective treatment.

While the use of bovine thrombin has been demonstrated to be an effective and relatively safe treatment, there remains some concern regarding long-term outcomes, including the low but potential risk of prion transmission and the risk of peri-procedural anaphylaxis. Therefore, the use of autologous thrombin for treatment of pseudoaneurysms has been explored. Initially described in a small group of patients by Quarmby et al, ${ }^{28}$ it has since been shown in larger trials to be highly effective and, in head-to-head comparisons with bovine thrombin, has shown equivalent results while being as much as $29 \%$ less expensive per treatment. ${ }^{29}$

Injection of cyanoacrylate glue has been demonstrated as a viable technique for management of visceral artery aneurysms and has since been shown to be safe and efficacious in small trials for femoral artery pseudoaneurysms after percutaneous access. ${ }^{30,31}$ However, opponents argue that such techniques are not as cost-effective and they may serve as a nidus for infection. Percutaneous coil embolization too has been demonstrated as a safe, effective alternative ${ }^{32}$ but is subject to similar criticism. Finally, a 2000 German study evaluated repair of postcatheterization pseudoaneurysms and arteriovenous fistulas with endovascular covered stents in the setting of failed compression therapy. They demonstrated a safe and effective technique; however, follow-up was limited ( $\sim 1$ year) and stent thrombosis was present in $17 \%$ of patients. Additionally, procedures are much more costly, invasive, and may be limited by anatomy. ${ }^{33}$

\section{Conclusion}

As demonstrated, femoral artery pseudoaneurysm after percutaneous access is a common complication and will continue to present itself as more endovascular procedures are performed. Duplex ultrasound will remain the diagnostic modality of choice as it is accurate, noninvasive, and costeffective. While ultrasound-guided compression has been demonstrated as a safe and effective therapy, it has significant limitations (most notably length of procedure and patient discomfort/intolerance). Thus, in the absence of a surgical indication, ultrasound-guided thrombin injection has been demonstrated to be an effective first-line treatment for iatrogenic femoral artery pseudoaneurysm with an excellent success rate of $97 \%$ and low rate of complications at $<1.3 \%$. $^{5}$

\section{Disclosure}

The authors report no conflicts of interest in this work.

\section{References}

1. Sambol EB, Mckinsey JF. Local complications: endovascular. In: Cronenwett J, Johnston W, editors. Rutherfords Vascular Surgery. 7th ed. Philadelphia, PA: Elsevier Health Sciences.

2. Chatterjee T, Do DD, Kaufmann U, Mahler F, Meier B. Ultrasoundguided compression repair for treatment of femoral artery pseudoaneurysm: acute and follow-up results. Cathet Cardiovasc Diagn. 1996;38(4):335-340.

3. Wixon CL, Philpott JM, Bogey WM, Powell CS. Duplex-directed thrombin injection as a method to treat femoral artery pseudoaneurysms. J Am Coll Surg. 1998;187(4):464-466.

4. Spies JB, Bakal CW, Burke DR, Cardella JF. Standard for diagnostic arteriography in adults. Standards of Practice Committee of the Society of Cardiovascular and Interventional Radiology. J Vasc Interv Radiol. 1993;4(3):385-395. 
5. Webber GW, Jang J, Gustavson S, Olin JW. Contemporary management of postcatheterization pseudoaneurysms. Circulation. 2007;115(20):2666-2674.

6. Katzenschlager R, Ugurluoglu A, Ahmadi A, et al. Incidence of pseudoaneurysm after diagnostic and therapeutic angiography. Radiology. 1995;195(2):463-466.

7. Hirano Y, Ikuta $\mathrm{S}$, Uehara $\mathrm{H}$, et al. Diagnosis of vascular complications at the puncture site after cardiac catheterization. $J$ Cardiol. 2004;43(6):295-265.

8. Helvie MA, Rubin JM, Silver TM, Kresowik TF. The distinction between femoral artery pseudoaneurysms and other causes of groin masses: value of duplex Doppler sonography. AJR Am J Roentgenol. 1988;150(5):1177-1180.

9. Lumsden AB, Miller JM, Kosinski AS, et al. A prospective evaluation of surgically treated groin complications following percutaneous cardiac procedures. Am Surg. 1994;60(2):132-137.

10. Toursarkissian B, Allen BT, Petrinec D, et al. Spontaneous closure of selected iatrogenic pseudoaneurysms and arteriovenous fistulae. J Vasc Surg. 1997;60(2):803-808.

11. Fellmeth BD, Roberts AC, Bookstein JJ, et al. Postangiographic femoral artery injuries: nonsurgical repair with US-guided compression. Radiology. 1991;178(3):671-675.

12. Kresowik TF, Khoury MD, Miller BV, et al. A prospective study of the incidence and natural history of femoral vascular complications after percutaneous transluminal coronary angioplasty. J Vasc Surg. 1991;13(2):328-333.

13. Schaub F, Theiss W, Busch R, Heinz M, Paschalidis M, Schomig A. Management of 219 consective cases of postcatheterization pseudianeurysm. J Am Coll Cardiol. 1997;30:670-675.

14. Cope C, Zeit R. Coagulation of aneurysms by direct percutaneous thrombin injection. AJR Am J Roentgenol. 1986;147(2):383-387.

15. Liau CS, Ho FM, Chen MF, Lee YT. Treatment of iatrogenic femoral artery pseudoaneurysm with percutaneous thrombin injection. $J$ Vasc Surg. 1997;26:18-23.

16. Calton WC, Franklin DP, Elmore JR, Han DC. Ultrasound-guided thrombin injection is a safe and durable treatment for femoral pseudoaneurysms. Vasc Surg. 2001;35(5):379-383.

17. Khoury M, Rebecca A, Greene K, et al. Duplex scanning-guided thrombin injection for the treatment of iatrogenic pseudoaneurysms. $J$ Vasc Surg. 2002;35(3):517-521.

18. Grewe PH, Mugge A, Germing A, et al. Occlusion of pseudoaneurysms using human or bovine thrombin using contrast-enhanced ultrasound guidance. Am J Cardiol. 2004;93(12):1540-1542.

19. Krueger K, Zaehringer M, Strohe D, Stuetzer H, Boecker J, Lackner K. Postcatheterization pseudoaneurysm: results of US-guided percutaneous thrombin injection in 240 patients. Radiology. 2005;236(3):1104-1110.
20. Schneider C, Malisius R, Kuchler R, et al. A prospective study on ultrasound-guided percutaneous thrombin injection for treatment of iatrogenic post-catheterisation femoral pseudoaneurysms. Int J Cardiol. 2009;131(3):356-361.

21. Liu Y-M, Hua Y, Liu Q, Ling C, Duan C. Efficacy comparison of different treatment methods for femoral pseudoaneurysms under ultrasonographic guidance. Chinese Journal of Cerebrovascular Diseases. 2006;3(10):433-436.

22. Stone PA, Campbell JE, Aburahma AF. Femoral pseudoaneurysms after percutaneous access. J Vasc Surg. 2014;30(5):1359-1366.

23. Samuels D, Orron DE, Kessler A, et al. Femoral artery pseudoaneurysm: Doppler sonographic features predictive for spontaneous thrombosis. J Clin Ultrasound. 1997;25(9):497-500.

24. Cox GS, Young JR, Gray BR, Grubb MW, Hertzer NR. Ultrasound-guided compression repair of postcatheterization pseudoaneurysms: results of treatment in one hundred cases. J Vasc Surg. 1994;19(4):683-686.

25. Sheiman RG, Mastromatteo M. Iatrogenic femoral pseudoaneurysms that are unresponsive to percutaneous thrombin injection: potential causes. AJR Am J Roentgenol. 2003;181(5):1301-1304.

26. Gehling G, Ledwig J, Schmidt A, Daniel WG, Werner D. Percutaneous occlusion of femoral artery pseudoaneurysm by para-aneurysmal saline injection. Catheter Cardiovasc Interv. 2003;58(4):500-504.

27. Finkelstein A, Bazan S, Halkin A, et al. Treatment of post-catheterization femoral artery pseudo-aneurysm with para-aneurysmal saline injection. Am J Cardiol. 2008;101(10):1418-1422.

28. Quarmby JW, Engelke C, Chitoloe A, Morgan RA. Autologous thrombin for treatment of pseudoaneurysms. Lancet. 2002;359(9310): 946-947.

29. Vazquez V, Reus M, Pinero A, et al. Human thrombin for treatment of pseudoaneurysms: cinoarusib of bovine and human thrombin sonogram-guided injection. AJR Am J Roentgenol. 2005;184(5): 1665-1671.

30. Mittal R, Stephen E, Keshava SN, Moses V, Agarwal S. Percutaneous cyanoacrylate glue embolization for peripheral pseudoaneurysms: an alternative treatment. Indian J Surg. 2012;74(6):483-485.

31. Aytekin C, Firat A, Gultekin B, Boyvat F, Tasdelen A. US-guided glue injection in the treatment of femoral pseudoaneurysms. Tani Girisim Radyol. 2003;9(2):257-259.

32. Kobeiter H, Lapeyre M, Becquemin JP, Mathieu D, Melliere D, Desgranges P. Percutaneous coil embolization of postcatheterization arterial femoral pseudoaneurysms. J Vasc Surg. 2002;36(1):127-131.

33. Thalhammer C, Kirchherr AA, Uhlich F, Waigand J, Gross CM. Postcatheterization pseudoaneurysms and arteriovenous fistulas: repair with percutaneous implantation of endovascular covered stents. Radiology. 2000;214(1):127-131
Journal of Vascular Diagnostics and Interventions

\section{Publish your work in this journal}

The Journal of Vascular Diagnostics and Interventions is an international, peer-reviewed journal of diagnostics, focusing on non invasive vascular investigation methods involved in the evaluation of vascular diseases. The journal is committed to the rapid publication in the fields of vascular diseases. Original research, review, case reports, expert opinion and commentaries are all considered for publication. The manuscript management system is completely online and includes a very quick and fair peer-review system, which is all easy to use. Visit http://www.dovepress.com/testimonials.php to read real quotes from published authors. 